\title{
Passive markers as a low-cost method of enriching cultural visits on user's demand
}

\author{
Anestis Koutsoudis, Fotis Arnaoutoglou, George Pavlidis* \\ Institute for Language and Speech Processing, 'Athena' Research and Innovation Centre, \\ University Campus at Kimmeria, PO BOX 159, Xanthi GR-67100, Greece \\ *Corresponding author E-mail: gpavlid@ceti.gr
}

Copyright $\odot 2014$ Anestis Koutsoudis, Fotis Arnaoutoglou, George Pavlidis. This is an open access article distributed under the Creative Commons Attribution License, which permits unrestricted use, distribution, and reproduction in any medium, provided the original work is properly cited.

\begin{abstract}
The idea of enriching a cultural heritage visitor's experience by employing mobile devices such as smartphones and tablets is an active research domain. This is mainly due to the worldwide user penetration of such devices along with their continuous hardware performance enhancement. This work presents the experience and feedback gained by one year of operation of an experimental information system that aims in enhancing the experience of a visitor on his/her demand. The evaluated system is based on the use case-scenario where the a mobile device is used to capture and decode passive markers (such as QR-codes) that are located on facades of selected important buildings in the old town of Xanthi, Greece, in order to enrich the visitor's experience through a broad context of historical and architectural information delivered over the Web.
\end{abstract}

\section{Introduction}

The enrichment of a cultural heritage visit experience through the exploitation of information technologies is an active R\&D field. Portable devices such as smartphones and tablets had a vast impact towards this direction. This is not only due to their extreme popularity (over one billion users according to a recent report from Strategy Analytics [1]) but also due to their continuous hardware and software advancements. Nowadays, it is common for such devices to be equipped not only with high-speed communication technologies but also with multi-core processors, graphics processing units, high resolution digital cameras, global-positioning circuitry, digital compasses and a variety of other sensors.

During the last decade, a number of research and development projects presented solutions that set a smartphone or tablet as a user-friendly terminal device that can enhance the cultural heritage visit experience. Today, it is obvious that such devices compose a versatile platform with an adaptable graphical user interface that can efficiently deliver multimedia content. This content can be generic or personalised. It can be presented either automatically by monitoring the user's actual position or on-demand based on the user's explicit request to interact with the system at a given time. Some of the most popular applications in the cultural heritage domain are focused on offering services such as information enrichment, interactive guides, augmented and virtual reality. Generally, the development of such systems is a vastly multilateral procedure due to the involvement of numerous challenges related to the characteristics of the environment being installed (indoors, outdoors or both), the distraction time of visitor's attention from the actual site or artefact, the visitor's effort and the non-uniformity of the system's user base (e.g. visitors of different skills, needs or interests).

In this paper, we discuss on the feedback gained by the one year operation and monitoring of an experimental low-cost cultural visit enrichment system that provides historical and architectural information about architectural entities (e.g. listed buildings, churches, squares, etc.) that are located in the centre of the old town of Xanthi. The current simplistic implementation delivers textual information to a mobile device on user's demand. A number of passive markers (QR- 
code plates) are used as triggers for initiating the delivery of information about a point of interest through a Web browser.

More specifically, in Section 2 we provide an overview of related cultural visit enhancement systems while in Sections 3 and 4 , we describe and objectively evaluate our experimental system based on its annual operation and we discuss the experience gained. We conclude in Section 5, which highlights the main properties and applicability of such systems in other cultural heritage domains.

\section{Related work}

Over the last years numerous cultural visit enrichment frameworks and systems have been proposed. They attempt to meet different requirements specifications that are dictated by the actual installation environment (e.g. museums, archaeological sites, urban areas, etc.). They also vary in the data types being used for content (e.g. textual information, images, video sequences, interactive/non-interactive multimedia content, etc.). On the other hand, they share engagement motives such as the efficient delivery of interesting information, the visitor's amusement, engagement and interaction with the environment that will lead to a significant visibility increase and understanding of our cultural heritage thesaurus [1]. In this section we present such systems that can be used for outdoor applications such as urban areas and archaeological sites and for indoor applications such as museum exhibitions, building interiors, etc.

Sintoris et al. [3] discussed on the idea of enabled spaces. As enabled, they define a space, which has the ability to use novel technologies and offer information services. Raptis et al. [4] performed a survey on the design of enabled space applications. In their work, they describe the importance of a theoretical framework that defines context through four dimensions (system, infrastructure, domain and physical) that complement and interact with each other. The Agamemnon project introduced the idea of automated monument recognition based on images captured by visitors using their mobile device. The system composes a dynamic multimedia guide system that takes under consideration the time scheduled for a visit [5]. Angelaccio et al. [6] proposed a system architecture that combines geographic information systems (GIS) and business intelligence in order to compose a platform useful for developing Websites that provide tourist information such as hotel reservations, price comparisons, reviews, etc. Brondi et al. [7] worked on the development of a markerless augmented reality framework oriented to the cultural heritage domain. The system exploits the inertial sensors and the GPS sensors of the device in order to transform it into an augmented reality device. Uk Im et al. [8] presented an audio guide system that exploits the GPS sensor in order to offer dramatized local stories or simple narratives that describe the history of a place. Stefanov et al. [9] have recently reviewed a number of free applications that provide information about historical and urban areas of cultural importance located in Bulgaria.

One the other hand, Mathew Nickerson discussed on using smartphones for audio-based guides in museums using the Voice-XML technology [10]. Kuusik et al. [11] presented a museum-oriented solution for personalised content access that addresses aspects such as unifying user preferences, context information and content annotation. Za et al. [12] presented the MID-blue framework, a multimedia information distribution system architecture that operates over Bluetooth communication technology. Their framework is able to provide specific content related to the user's position within the premises of a museum. Furthermore, Rung et al. [13] created an iOS-based application focused on the work of Henri de Toulouse-Lautrec that could be used before, during or after the visit to the exhibition. A museum guide system was also presented by Ruf et al. [14]. Their system was based on the calculation of the scale-invariant feature transform (SIFT) on photos captured by a smartphone. Using SIFT coefficients as a content descriptor the system could recognise paintings in an art gallery and retrieve relevant information. Choudary et al. [15] presented an augmented reality system that is focused on prehistoric cave engravings. The system augments the captured images with expert's drawings, highlighting in real time the animal engraving that are almost impossible to observe with the naked eye. In order to track the drawings the systems detects a custom type of passive markers located around the engravings. Furthermore, the idea of using passive markers such as QR-codes has already been exploited for both indoors and outdoors applications [16]-[21]. It is a cost-effective and powerful technology to detect the user's context and to infer user interests [20]. The outdoor experimental system evaluated in this work also exploits QR-codes. One of the leading motives behind its implementation was to acquire an objective feedback of such a system's installation and operation while based on a real-world use-case scenario. Such feedback can be considered as a sufficient basis for future investments in installing such information systems or extending their functionality.

\section{System architecture and implementation}

The main idea behind the system described in this work is that a mobile device delivers historical and architectural information about architectural entities through a Web-browser according to the visitor's demand. The system does not offer a strict follow-specific-path interactive guide. It is a more open solution where the visitor chooses his/her own path to follow and when a point-of-interest (POI) captures his/her attention then through a minimised number of user 
interactions, he/she can have access the digital content over the Web. QR-codes and generally passive markers based systems offer accurate localisation of the visitor through inexpensive infrastructure.

In addition, the cost of the presented system remains low as the required capabilities are already built in the visitor's device. More specifically, the visitor can use any mobile device that is: $i$ ) equipped with a digital camera, ii) can access the Web over WiFi or over a mobile carrier, iii) has any Web browser and QR-code decoding software installed. In fact, all major operating systems provide such functionality in terms of pre-installed software or as free applications that can be downloaded through a secured application download service (e.g. Google play, App store, etc.). Thus, the development was related solely on the implementation of the system's backend.

Fig. 1 illustrates the simplistic system's architecture along with the steps of user interaction involved. The backend operates on PHP, MySQL and CSS3 technologies and is able to deliver information using URI-based queries that include parameters that uniquely identify POIs and preferred textual content language. The system replies to each query with a Web page, which content is adapted to the screen resolution of the target device. Each Web page contains a generic text of historical and architectural aspects of the POI, followed by a number of related references.

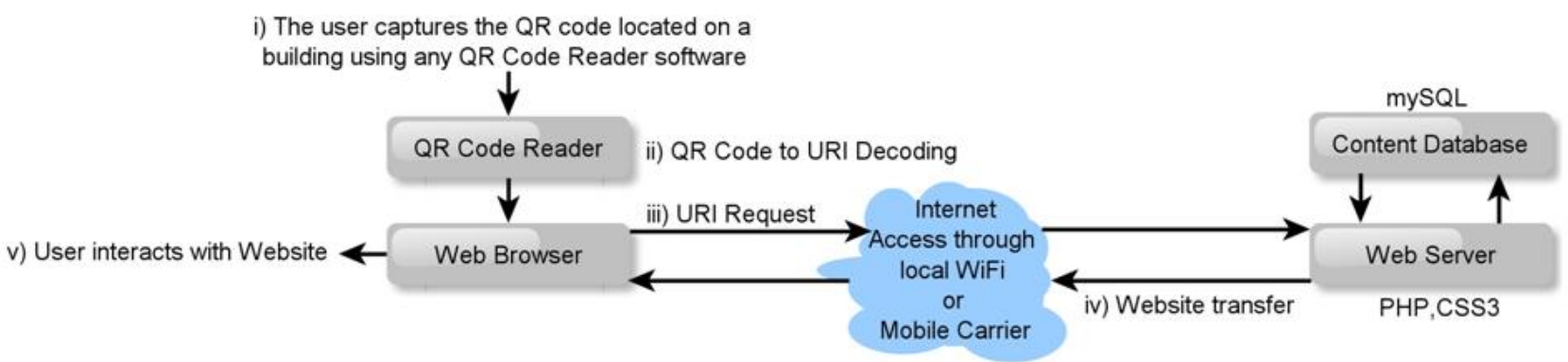

Fig. 1: System architecture and steps of user interaction

Furthermore, the centre of the old town of Xanthi, Greece was selected for installing and testing the system not only for its cultural importance but also because it has visiting activity throughout the year and because it is covered by the municipality's free Wi-Fi network. A total of thirteen POIs were identified and documented. Each of the POIs is referred to an architectural entity such as a listed building, a church or even a square of historic importance. In addition, all POIs are located in the centre of the old town as it is considered the most common region to be reached by visitors.

Additionally, all URI-based queries, that trigger the system to reply with a Web page, where encoded into QR-code images and were printed on custom metal plates covered with an antiglare film to improve camera capturing robustness. They were placed either on the facades of the buildings or on other high-visibility spots. Special permissions had to be acquired by the owners of the buildings and the municipality of Xanthi. Each plate has the equivalent size of an $A 4$ page and carries a short bilingual explanatory text about using the system. Fig. 2 depicts the POIs dense spatial distribution over an aerial view of the old town's centre along with a picture of an installed plate.

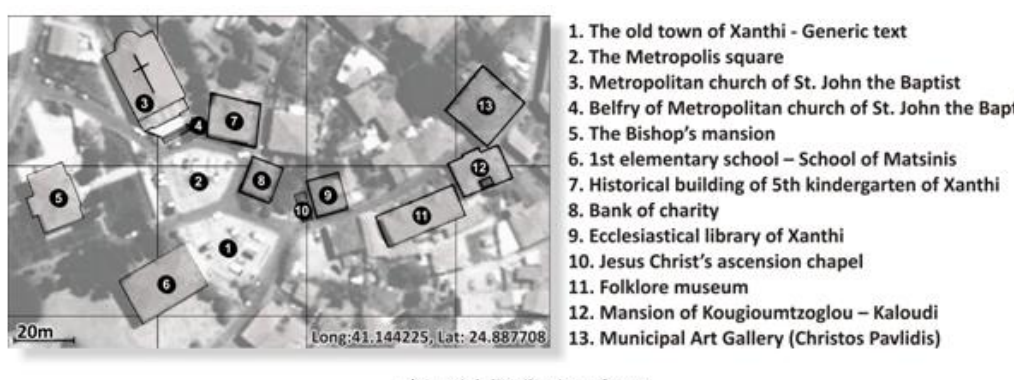

a) Spatial distribution of POls

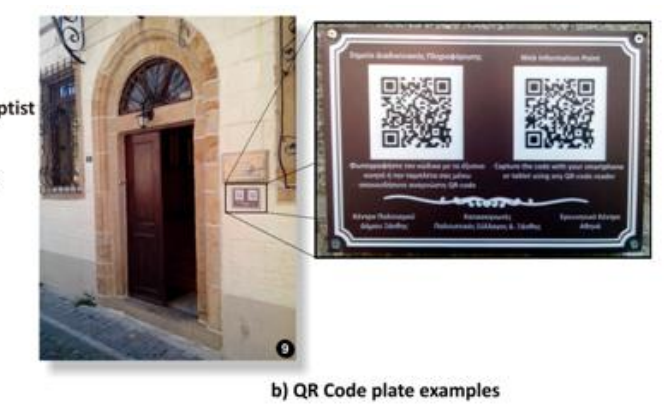

b) QR Code plate examples

Fig. 2: POIs spatial distribution and QR-code plate examples

\section{System evaluation}

In order to perform an objective evaluation of the experimental system, we monitored its annual usage based on mechanisms offered by the system's Web server and gathered quantitative data. In this section, we present some of the 
statistics related to its operation. Overall evaluation results covering a full year of operation are shown in Fig. 3, which depicts visits per POI and language, per month, day and hour of day, along with data regarding the operating system used by the visitors' devices. In the following paragraphs we provide comments and insight on these results.

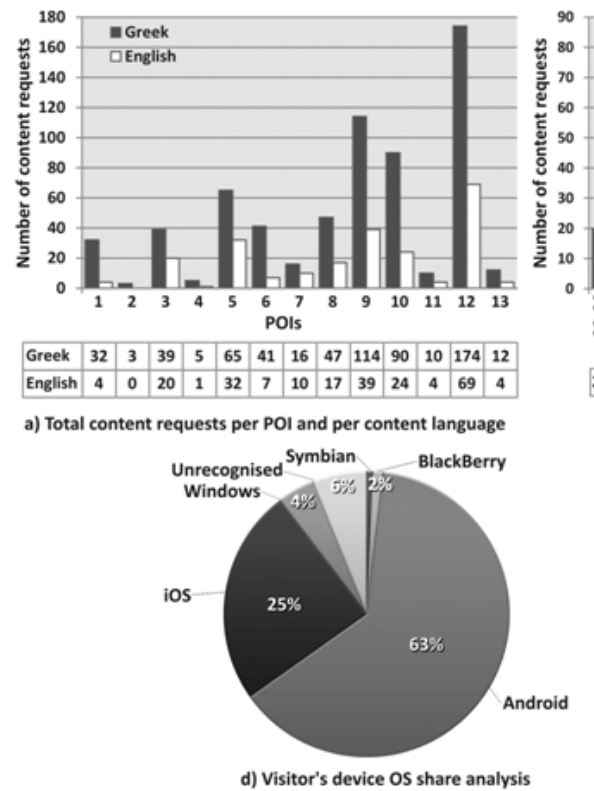

d) Visitor's device OS share analysis

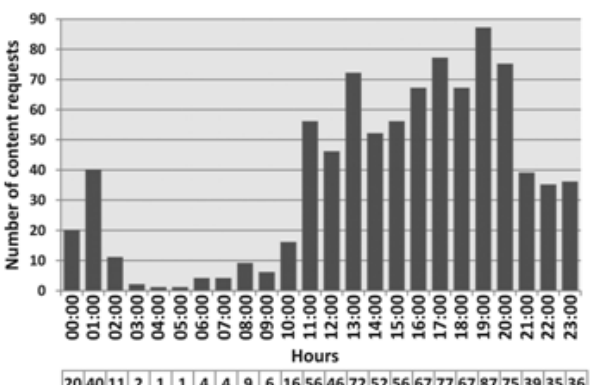

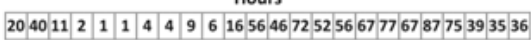

b) Total content requests per hour

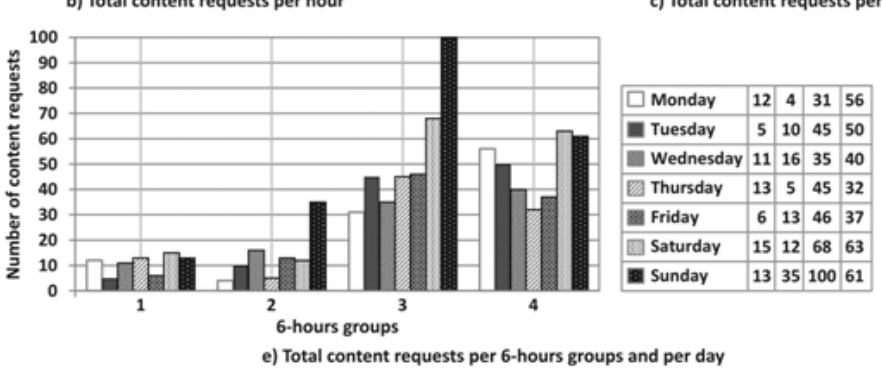

Fig. 3: Statistical analysis of system's annual usage

Fig. 3a depicts the totals of content requests (unique hits) served by the system ordered per POI and per content language. A total of 879 unique content requests were served by the system ( 648 requests for Greek language content and 231 requests for English language content). The higher number of requests for the Greek language content may be considered as an indicator of the old town visitors' nationality. Fig. 3a clearly depicts that there are a few favoured POIs regardless of the content's language. This is highly related to specific POI's properties such as the notable architectural characteristics and the visitor's easiness of accessing the passive marker. Considering the case where most visitors walk on the middle of a pedestrianized street to have a good view of the buildings of both sides then it is probable that a marker that is located in narrow streets (POIs 9 and 10) or on a crossroad (POI 12) will be more popular. Again, POI 12 is located on a crossroad that it considered as one of the most photographed areas of the old town and this is also depicted by its high number of views. It should be noted that POIs 2 and 4 were damaged at some point within the first month of the system's operation. The plate at POI 2 was completely removed while POI 4 content was scratched to a degree that QR-codes were unreadable. Nevertheless, the damaged plates $(\approx 15 \%$ of total number of plates) were not replaced before the completion of the experiment, as vandalism was also an important aspect that should be taken under consideration during the experimental operation of such an unattended system.

Fig. $3 b$ illustrates the totals of content requests per hour. The system is found to be used even on late-night hours. Fig. $3 \mathrm{c}$ depicts the system's total usage activity per month. The highest numbers of content requests are found during the months August and October (112 and 125 respectively). It should be noted that during the last week of August and first week of September an annual festival dedicated to the old town takes place and thus the number of visitors is significantly increased.

Fig. $3 \mathrm{~d}$ depicts the percentage share of the visitor's mobile device operating system. The results gained do step with the current generic user penetration levels of these operating systems. The Android operating system was the most popular between the visitors' devices followed by iOS. The share ambiguity of the unrecognised operating systems devices was a result of the inability or denial of the mobile device's Web browser to provide the logger script with the request information. The wide range of operating systems portrays the system's high accessibility from different mobile devices. It should be noted that the version fragmentation of the Android operating system that is depicted in the system's $\log$ (Version 2.2.1 up to more recent such as version 4.1.2) also confirms the previous conclusion.

Fig. 3e illustrates the total activity of the system on a daily basis. The totals are organised into four groups where each one represents a time period of six hours (00:00-05:00,06:00-11:00, 12:00-17:00 and 18:00-23:00). The weekend days are found to be the most active ones as well as midday and evening hours. 
Other performance aspects such as the system's responsiveness was unable to be accurately monitored as the primary bottleneck, that is the variable wireless data transfer bandwidth, relies on the current Wi-Fi network or on the bandwidth offered by the various mobile carriers. Nevertheless, the small data size $(\approx 30 K B)$ of each POI content resulted a lowcost in terms of usage system (mobile carrier data transfer charges) and an average delay of eight seconds for the procedure of QR-code capturing, decoding and content transferring using a $3 \mathrm{G}$ network of a national mobile carrier. The previous measurements were performed using a single core (Cortex A8-1Ghz) Android (version 4.0.3) smartphone device equipped with the free QR Droid [22] application and Android's native Web browser. Last but not least, it is worth to mention that a positive side effect of such systems is that content retrieval accuracy and precise visitor localisation is always guaranteed by the use of unique passive markers.

The previous statistics indicate that visitors were familiar with passive marker based systems and were interested in either getting additional information or curious to use their mobile devices for purposes other than the ordinary. It should be noted though that although the old town of Xanthi is covered by a Wi-Fi network that can be used for free it requires a registration procedure that might be considered by some visitors as a time consuming or distracting procedure. Moreover, in cases where a visitor has to use a mobile carrier to access the Web, the significant issue of data transfer charges is raised especially for those that are using roaming services.

\section{Conclusion}

Nowadays, visiting experience enhancements can be achieved with the use of a familiar to the visitor personal device. In this work, we presented the feedback gained after the annual operation of an experimental low-cost passive markers based information system that can be accessed by a wide range of mobile devices. We based our case study in an urban area of historical importance and architectural multiformity, where the visitor could retrieve information on-demand over the Web. The obtained statistics indicate that the existence of such a system is meaningful and it will be used up to some extent to enhance the visiting experience while keeping the cost of infrastructures and operation to a minimum. Another important characteristic of the development costs had to do with use of the mobile device's build-in capabilities. Such an approach allows the minimisation of software development. Again, the low maintenance cost is highly associated with the passive markers. They have key properties such as their construction quality and durability that should be taken under consideration. The use of an antiglare film proved to be an efficient solution for outdoor applications that experience varying lighting conditions. Furthermore, the large passive marker size (equivalent to an $A 4$ page) used in this experiment had to do with the need to make the existence of the system obvious. In a real case scenario, small sized passive markers can be used, as those will be supported by other means of publicity. Conclusively, passive marker-based systems are cost-efficient solutions that should be taken under consideration in cases where there is a need to create inexpensive and discreet outdoor or indoor information systems. The usage statistics presented in this work play an objective indicative role for the development of similar systems. Yet, the coverage and the quality of the content provided by such systems do play a significant role in their overall success. We will be using the experience and feedback gained from this work as a basis for the future development of a similar system of extended functionality that will be also related to semi-automated guided tours and other topological-based services by exploiting previous works [23].

\section{Acknowledgements}

The authors would like to thank Xanthi's cultural association 'Kataskinotes' ('Campers'), Xanthi's Cultural Centre and the Cultural Heritage Research Group of the Institute for Language and Speech Processing of 'Athena' Research and Innovation Centre for their support in the implementation and installation of the experimental system.

\section{References}

[1] Strategy Analytics, available online: http://www.strategyanalytics.com/default.aspx?mod=saservice\&a0=91\#0

[2] N. Ryan, P. Mohr, D. Manzaroli, G. Mantovani, S. Bartolini, A. D’Elia, M. Pettinari, L. Roffia, L. Sklenar, F. Garzotto, T. Salmon, "Interoperable multimedia mobile services for cultural heritage sites", Proceedings of EPOCH Open Digital Cultural Heritage Systems, Rome, Italy, February 2008.

[3] C. Sintoris, D. Raptis, A. Stoica, N. Avouris, "Delivering multimedia content in enabled cultural spaces", Proceedings of the $3^{\text {rd }}$ International Mobile Multimedia Communications Conference, Nafpaktos, Greece, 27-29 August, 2007.

[4] D. Raptis, N. Tselios, N. Avouris, "Context-based design of mobile application for museums: A survey of existing practices", Proceedings of the $7^{\text {th }}$ International Conference on Human Computer Interaction with mobile devices and services, Salzburg, Austria,19-22 September, 2005, pp. $153-160$.

[5] M. Ancona, M. Cappello, M. Casamassima, W. Cazzola, D. Conte, M. Pittore, G. Quercini, N. Scagliola, M. Villa, "Mobile vision and cultural heritage: the Agamemnon project”, Proceedings of $1^{\text {st }}$ International Workshop on Mobile Vision, Graz, Austria, May 2006, pp. 1-15.

[6] M. Angelaccio, A. Basili, B. Buttarazzi, "Using Geo-Business intelligence and social integration for smart tourism cultural heritage platforms", Proceedings of $22^{\text {nd }}$ IEEE International Workshop on Enabling Technologies: Infrastructure for Collaborative Enterprises, Hammamet, Tunisia, 17-20 June, 2013, pp.196-199. 
[7] R. Brondi, M. Carrozzino, F. Tecchia, M. Bergamasco, "Mobile augmented reality for cultural dissemination", Proceedings of $1^{\text {st }}$ International Conference on Information Technologies for Performing Arts, Media Access and Entertainment, Firenze, Italy, 7-9 May, 2012, pp.113-117.

[8] D. Uk Im, H. Ro Yoon, J. Yeong Lee, "A case study of the walking trail applications on GPS-based smartphone using the local narrative", Advanced Science and Technology Letters, 12 (2012) 100-106.

[9] T. Stefanov, M. Stefanova, "Mobile applications for cultural and historical places", Digital Presentation and Preservation of Cultural and Scientific Heritage, 3 (2013) 227-234.

[10] M. Nickerson, "All the world is a museum: Access to cultural heritage information anytime, anywhere", Proceedings of ICHIM 2005 - Digital Culture \& Heritage, Paris France, 21-23 September, pp. 2-15.

[11] A. Kuusik, S. Roche, F. Weis, "SMARTMUSEUM: Cultural content recommendation system for mobile users", Proceedings of $4^{\text {th }}$ International Conference on Computer Sciences and Convergence Information Technology, Seoul, South Korea, 24-26 November, 2009, pp. 477-482.

[12] S. Za, E. D’Atri, F. Mazzei, A. M. Braccini, "Multimedia contents provision using Bluetooth for cultural heritage applications: the MID-Blue project", Proceedings of $4^{\text {th }}$ Interop-Vlab.It Workshop on Pervasive Computing for Networked Enterprises, Rome, Italy, October 6-7, 2011, pp. 9-16.

[13] M. H. Rung, D. Laursen, "Adding to the experience: Use of smartphone applications by museum visitors", Proceedings of the Transformative Museum Conference, Roskilde, Denmark, 23-25 May, 2012, pp. 314-324.

[14] B. Ruf, E. Kokiopoulou, M. Detyniecki, "Mobile museum guide based on fast SIFT recognition", Proceedings of $6^{\text {th }}$ International Workshop on Adaptive Multimedia Retrieval, Berlin, Germany, 26-27 June, 2008, pp. 170-183.

[15] O. Choudary, V. Charvillat, R. Grigoras, P. Gurdjor, "MARCH: Mobile augmented reality for cultural heritage", Proceedings of $17^{\text {th }}$ ACM International Conference on Multimedia, Beijing, China, 19-24 October, 2009, pp.1023-1024

[16] Explore Amsterdam with QR-codes, available online: http://www.iamsterdam.com/en-GB/experience/about-amsterdam/areas/amsterdamneighbourhoods/explore-amsterdam-qr-codes

[17] Wikipedia City, http://blog.wikimedia.org/2012/05/16/monmouthpedia_day

[18] Annette Haworth, Peter Williams, "Using QR-codes to aid accessibility in a museum", J Assistive Technologies, 6 (2012) 285-291.

[19] U. B. Ceipidor, C. M. Medaglia, A. Perrone, M. De Marsico, G. Di Romano, "A museum mobile game for children using QR-codes", Proceedings of the $8^{\text {th }}$ International Conference on Interaction Design and Children, Como, Italy, 2009, pp. $282-283$.

[20] S. Andolina, D. Pirrone, G. Russo, S. Sorce, A. Gentile, "Exploitation of mobile access to context-based information in cultural heritage fruition", Proceedings of the $7^{\text {th }}$ International Conference on Broadband, Wireless Computing, Communication and Applications, Victoria, Canada, 12-14 November, 2012, pp.322-328.

[21] Monmouth, the world's first Wikipedia Town, available online: http://blog.wikimedia.org/2012/05/16/monmouthpedia day

[22] QR Droid application, DroidLa, available online: https://play.google.com/store/apps/details?id=la.droid.qr\&hl=en

[23] A. Koutsoudis, K. Stavroglou, G. Pavlidis, C. Chamzas, "3DSSE - A 3D Scene Search Engine, Exploring 3D Scenes Using Keywords", J Cultural Heritage 13 (2012) 187-194. 